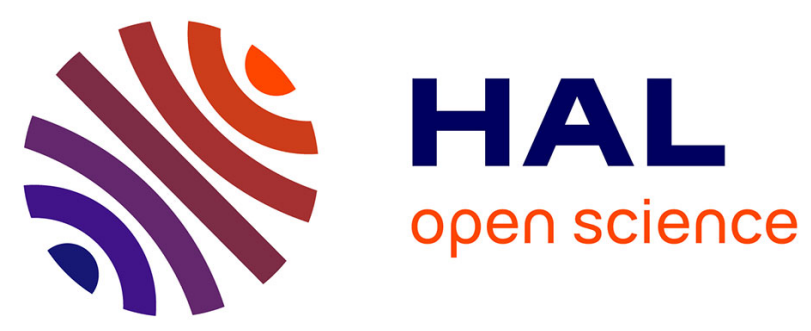

\title{
Nonlinear acoustic propagation in bubbly liquids: Multiple scattering, softening and hardening phenomena
}

Jean-Baptiste Doc, Jean-Marc Conoir, Régis Marchiano, Daniel Fuster

\section{To cite this version:}

Jean-Baptiste Doc, Jean-Marc Conoir, Régis Marchiano, Daniel Fuster. Nonlinear acoustic propagation in bubbly liquids: Multiple scattering, softening and hardening phenomena. Journal of the Acoustical Society of America, 2016, 139 (4), pp.1703-1712. 10.1121/1.4945452 . hal-01312438

\section{HAL Id: hal-01312438 \\ https://hal.science/hal-01312438}

Submitted on 6 May 2016

HAL is a multi-disciplinary open access archive for the deposit and dissemination of scientific research documents, whether they are published or not. The documents may come from teaching and research institutions in France or abroad, or from public or private research centers.
L'archive ouverte pluridisciplinaire HAL, est destinée au dépôt et à la diffusion de documents scientifiques de niveau recherche, publiés ou non, émanant des établissements d'enseignement et de recherche français ou étrangers, des laboratoires publics ou privés. 


\title{
Nonlinear acoustic propagation in bubbly liquids: multiple scattering, softening and hardening phenomena
}

\author{
Jean-Baptiste Doc, Jean-Marc Conoir, Régis Marchiano and Daniel Fuster \\ Sorbonne Universités, UPMC Université Paris 06, CNRS UMR 7190, Institut Jean Le Rond d'Alembert, \\ F-75005 Paris, France
}

\begin{abstract}
The weakly nonlinear propagation of acoustic waves in monodisperse bubbly liquids is investigate numerically. A hydrodynamic model based on the averaged two-phase fluid equations is coupled with the Rayleigh-Plesset equation to model the dynamics of bubbles at the local scale. The present model is validated in the linear regime by comparing with the Foldy approximation. The analysis of the pressure signals in the linear regime highlights two resonance frequencies: the Minnaert frequency and a multiple scattering resonance which strongly depends on the bubble concentration. For weakly nonlinear regimes, the generation of higher harmonics is observed only for the Minnaert frequency. Linear combinations between the Minnaert harmonics and the multiple scattering resonance are also observed. However, the most significant effect observed is the appearance of softening-hardening effects that share some similarities with those observed for sandstones or cracked materials. These effects are related to the multiple scattering resonance. Downward or upward resonance frequency shifts can be observed depending on the characteristic of the incident wave when increasing the excitation amplitude. We show that the frequency shift can be explained assuming that the acoustic wave velocity depends on a law different from those usually encountered for sandstones or cracked materials.
\end{abstract}

\section{Introduction}

The nonlinear propagation in bubbly liquids is an attractive theoretical problem combining various mechanisms that can make the system's response nonlinear. On one hand, the acoustic waves can by themselves lead to shock waves in a liquid. On the other hand, bubbles are known to have a highly nonlinear response that influences the propagation of waves at large scales. From an application point of view, the interaction between nonlinear acoustic waves and bubbles finds application in various fields. In medical imaging, the use of ultrasound contrast agents (UCAs) has significantly increased the quality of imaging techniques $1,2,3,4$. In nondestructive evaluation, the nonlinear propagation of acoustic waves has been proposed to determine histogram of bubble population inside new generation of nuclear plants [5].

Bubbly liquids can be considered as acoustic metamaterials where bubbles play the role of strong scatters that exhibit bandgap arising from their low-frequency resonance 6]. Different phenomena can modify the scattered properties of the medium. For instance, bubbles (or contrast agents) can accumulate due to the presence of walls reinforcing multiple scattering effects [7. These interactions are relatively well understood in the linear regime where they are generally well described by the Foldy's approximation [8, 9, 10, however, for moderate forcings, the interaction between the multiple scattering induced by the bubbles and the presence of nonlinear effects is less intensively investigated.

Based on the amplitude of the incident acoustic wave we identify three regimes of propagation. In the linear regime, which applies for small amplitude perturbations, the simulation of bubble clusters dates back to Foldy in 1945 [8] who presents a general multiple scattering theory applied later to bubbles 9, 10]. When the amplitude of the incident wave is large it is possible to identify another regime where shock waves naturally develop in the system. In these situations, one needs to resort to models as those proposed by Zhang and Prosperetti [11] who derive averaged equations for disperse two phase flows that are known as "ensemble averaged equations". More recently, Ando et al. [12] (see references therein) have presented simulations of shock waves in polydispersed bubbly flows. Finally, between the linear regime and the shock wave propagation regime, it is possible to define an intermediate regime where weakly nonlinear propagation effects occur. A number of models for nonlinear multiple scattering in liquids containing free bubbles have been derived. In 1968, Van Wijngaarden [13 presented a heuristic model that was later improved by Caflisch et al. 14] and Commander and Prosperetti [10] to include an effective equation of state for the gas. Other models based on Van Wijngaarden-Cafisch treatment include the works of [4, 15]. It is also worth mentioning the summary of models included in Hamilton \& Blackstock [16], where the pioneering works of Zabolotskaya et al. are recalled. Using a wave equation coupled with the Rayleigh-Plesset equation, these models are capable to capture the influence of bubbles on the nonlinear propagation properties of waves through a coefficient of nonlinearity. The appearance of harmonics due to the nonlinear dynamics of bubbles is the main phenomenon observed.

In this manuscript we focus our attention on the numerical analysis of the propagation of acoustical pulses with moderate amplitudes in a bubbly liquid where the source of non-linearity in the system can be only attributed to the nonlinear response of the bubbles. Section II is devoted to the derivation of the model equations. Then, the numerical method used to solve the problem is presented in Section III. To assess the ability of the numerical scheme to reproduce the multiple scattering effects, we show a comparison between numerical results and the linear theory in Section IV where we also introduce multiple scattering analysis techniques to better characterize the response of the system. Based on these analyses, in Section $\mathrm{V}$ we investigate the interaction between multiple scattering effects and nonlinear propagation by increasing the amplitude of the incident wave. Finally, the classical effects of harmonic generation is revisited by using the results of the nonlinear multiple scattering.

\section{Hydrodynamic model}

Many physical phenomena can play a role on bubble dynamic response and the propagation of waves through bubbly liquids: heat and mass transfer effects [17, direct bubble-bubble interactions, fragmentation, or polydispersity are some examples of complex processes that should be correctly modeled for real applications [18, 19, 20]. Here, in order to investigate the influence of weakly nonlinear bubble oscillations on the propagation of waves, we choose the simplest model able to reproduce these effects. In particular this work is based 
on a classical hydrodynamic model equivalent to that of Zabolotskaya et al [16. This model, used for instance in 15, is a simplification of the general formulation presented by Zhang and Prosperetti 11 and already captures the essential mechanisms that we want to investigate in this manuscript. Just to summarize, the acoustic behavior of bubble suspension in the ambient fluid is described by homogeneous and averaged two-phase fluid equations. The use of an homogeneous model implies that the acoustic wavelenght is much larger than the bubble radius. Bubbles are assumed to be randomly and homogeneously distributed in the mixture. Hydrodynamic forces, responsible of the translational motion, exerted by the ambient-fluid on the bubbles (Faxen forces) are neglected The ambient fluid is assumed to be compressible with specific mass $\rho$, pressure $p$ and sound speed $c$. All the nonlinear terms present in the large scale wave equations are neglected except the source term capturing the influence of the bubble oscillation at large scales. For all quantities, index 0 is used to designate their value at static equilibrium. Similarly to Hamilton [16] and references therein, the propagation in bubbly medium is described by the following coupled set of equations:

$$
\left\{\begin{array}{l}
\left(1-\Phi_{0}\right)\left[\frac{1}{c_{0}^{2}} \frac{\partial p}{\partial t}+\rho_{0} \frac{\partial v}{\partial x}\right]=\rho_{0} n_{0} \frac{\partial V_{b}}{\partial t}, \\
\left(1-\Phi_{0}\right) \rho_{0} \frac{\partial v}{\partial t}+\frac{\partial p}{\partial x}=0
\end{array}\right.
$$

where $V_{b}$ is the bubble volume and $\Phi_{0}$ is the volume fraction of the bubble within the total suspension volume. The bubble cloud is assumed to be monodisperse.

In the following the bubble dynamic response is governed by the Rayleigh-Plesset Noltingk Neppiras and Poritsky (RPNNP) equation 21. Because we are going to investigate high frequency effects appearing above the Minnaert frequency, we decide to neglect thermal effects. Bubbles are assumed to contain an inmiscible gas, hence no mass exchange occurs between the bubble and the ambient fluid. The influence of the bubble dynamics on the wave propagation is described by the right term of Eq. 1a, where the motion of an incompressible spherical bubble is described by the classical RPNNP equation:

$$
\rho_{0}\left(R \ddot{R}+\frac{3}{2} \dot{R}^{2}\right)=P_{g e}\left(\frac{R_{0}}{R}\right)^{3 \kappa}-\frac{2 \sigma}{R}-4 \mu \frac{\dot{R}}{R}-\left(P_{0}+p\right),
$$

where $\sigma$ denotes the surface tension, $P_{0}$ and $R_{0}$ are the static pressure and the equilibrium bubble radius at rest respectively and $P_{g e}=P_{0}+2 \sigma / R_{0}$ is the initial pressure inside the bubble. Note that the bubble internal pressure is assumed uniform and given by the polytropic law. Although the fluid viscosity, $\mu$ is neglected at the large scales, viscous effects are retained in Rayleigh-Plesset equation to model the bubble dynamic response.

The values of the mechanical parameters used with the model are presented in table 1

\begin{tabular}{|c|c|}
\hline$\rho_{0}$ & $1000 \mathrm{~kg} . \mathrm{m}^{-3}$ \\
\hline$c_{0}$ & $1500 \mathrm{~m} \cdot \mathrm{s}^{-1}$ \\
\hline$\sigma$ & $0.075 \mathrm{~N} . \mathrm{m}^{-1}$ \\
\hline$\mu$ & $10^{-3} \mathrm{~Pa} . \mathrm{s}$ \\
\hline$\kappa$ & 1 \\
\hline$P_{0}$ & $1 \mathrm{~atm}$ \\
\hline
\end{tabular}

Table 1: Values of the mechanical parameters used for all numerical results for the liquid and bubbly phases.

\section{Numerical method}

The system of equations (1) is solved numerically by a finitedifference scheme. To ensure stability of the numerical resolution, a variable transformation is used to normalize the mean velocity and pressure fields. By posing the relations $P=p / P_{0}$ and $Q=v \rho_{0} c_{0} / P_{0}$, Eq. (1) can be rewritten as follows:

$$
\left\{\begin{array}{l}
\frac{\partial P}{\partial t}=-c_{0} \frac{\partial Q}{\partial x}+\frac{c_{0}^{2}}{\left(1-\Phi_{0}\right) P_{0}}\left(\rho_{0} n_{0} \frac{\partial V_{b}}{\partial t}\right) \\
\frac{\partial Q}{\partial t}=-\frac{c_{0}}{\left(1-\Phi_{0}\right)} \frac{\partial P}{\partial x}
\end{array}\right.
$$

where $n_{0}=\frac{\Phi_{0}}{4 / 3 \pi R_{0} 3}$ according to the monodisperse approximation of the bubbles into the suspension.

The two coupled transport equations (3) are discretized by a Leap-Frog scheme. The variation of the bubble volume can be expressed: $\partial V_{b} / \partial t=4 \pi \dot{R} R^{2}$. The Rayleigh-Plesset equation (2) is solved locally by an explicit Runge-Kutta method of order 4 on each time step. An adaptive step size is used in order to correctly describe the fast variation of bubble radius. Then, the following marching algorithm is obtained:

$$
\begin{gathered}
P_{x}^{t+\Delta t}=P_{x}^{t-\Delta t}-c_{0} \frac{\Delta t}{\Delta x}\left(Q_{x+\Delta x}^{t}-Q_{x-\Delta x}^{t}\right)+2 \Delta t M_{x}^{t} \\
Q_{x}^{t+\Delta t}=Q_{x}^{t-\Delta t}-\frac{c_{0}}{\left(1-\phi_{0}\right)} \frac{\Delta t}{\Delta x}\left(P_{x+\Delta x}^{t}-P_{x-\Delta x}^{t}\right)
\end{gathered}
$$

with

$$
M_{x}^{t}=\frac{3 \rho_{0} c_{0}^{2}}{R_{0}{ }^{3} P_{0}} \frac{\Phi_{0}}{\left(1-\Phi_{0}\right)} \dot{R}_{x}^{t} R_{x}^{t^{2}} .
$$

The time and space steps are represented by $\Delta t$ and $\Delta x$ respectively. The CFL condition is defined in reference to the sound speed in the fluid phase as CFL $=c_{0} \Delta t / \Delta x$. The indexes $t$ and $x$ indicate the position in time and space in the marching scheme. A Lax-Wendroff scheme is used to initialize the numerical resolution of the hydrodynamical model.

To study the wave dispersion in bubbly liquids, a Gaussian pulse (see Fig. 1) is imposed for the initial pressure disturbance as follows:

$$
P(x)=A e^{-x^{2} / h^{2}} \quad \text { at } t=0,
$$

where $A$ and $h$ respectively represent the amplitude and the standard deviation of the Gaussian function. Thus, the equivalent wavelength used for the sizing of the numerical domain is defined as the full width at half maximum of the Gaussian peak, according to $\lambda_{0}=2 h \sqrt{2 \ln 2}$. The initial mean velocity field of the fluid is set to zero. The initial bubble radius is then obtained from Laplace's equation, so that bubbles are in equilibrium with the initial pressure. The initial bubble velocity is set to zero.

Perfectly Matched Layers 22] are used to bound the numerical domain (see Fig. 1). Bubbles are considered in the PML layers to avoid the generation of reflection waves at the interface between physical and absorbent mediums. From upstream to downstream to the PML layers, the viscous coefficient $\mu$ is progressively increasing to locally limit the acoustical contribution by bubbles. 


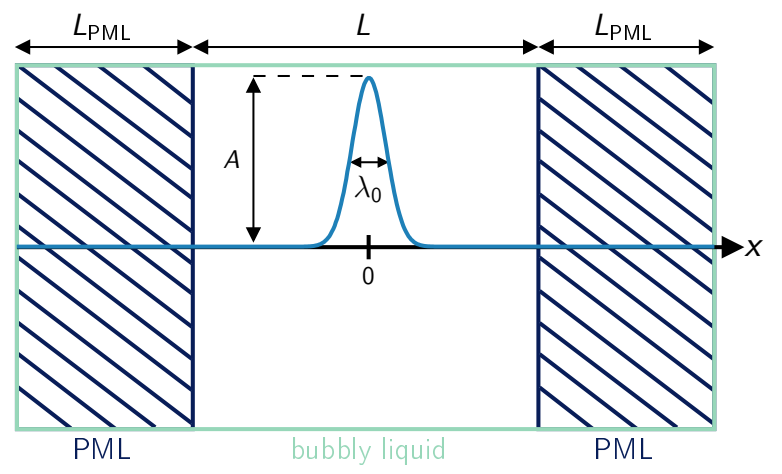

Fig. 1: Schematic of the numerical domain with representation of the initial pressure disturbance.

\section{Linear wave propagation in bubbly liquids}

\subsection{Comparison with the ECAH theory}

In this section we start validating the numerical model by comparing the phase velocity and attenuation computed from the model presented above with the ECAH multiple scattering theory 23. ECAH theory is based on the Foldy approximation 8 for dilute suspension and it can also be derived from the quasi-crystalline approximation [23]. More details on the analogy between EACH theory and the hydrodynamic model used in this study is given in [24].

Because the ECAH theory is only applicable for linear wave propagation, we simulate the response of an initial pressure disturbance with an initial Gaussian shape with amplitude equal to $A=10^{-4} P_{0}$ and standard deviation $h=10 R_{0}$. The numerical simulations contained in this section are carried out for bubbles of static radius $R_{0}=10^{-5} \mathrm{~m}$. The spatial step size is set to $\Delta x=\lambda_{0} / 50$ and the CFL condition is equal to 0.01 . The numerical domain is sized by $L=30 \lambda_{0}$ and $L_{\mathrm{PML}}=85 \lambda_{0}$ (see Fig. 1).

Fig. 2 shows how the Gaussian pressure pulse is dispersed after propagating through the bubbly liquid. As expected, high-frequency waves propagate more quickly than lowfrequency waves. This is a well-known behaviour already observed numerically and experimentally [12, 20, 25]. By comparing two different time steps, one can observe low frequency oscillations due to the local effect of the bubble dynamics (typically on the interval $x=[0,2] \mathrm{mm}$ ). High-frequency waves propagate approximately at the sonic speed of water alone.

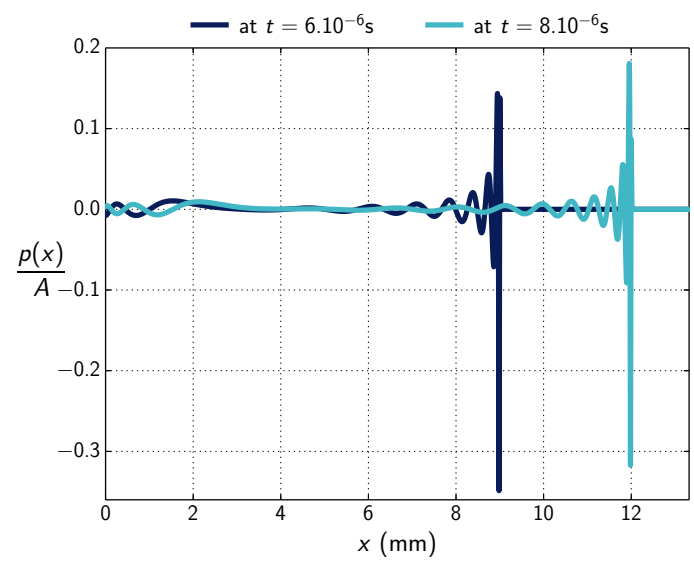

Fig. 2: Spatial evolution of the averaged acoustic pressure at two different time step. $\left(A=10^{-4} P_{0}, \Phi_{0}=1 \%, R_{0}=10^{-5}\right.$ and $\left.h=R_{0}\right)$

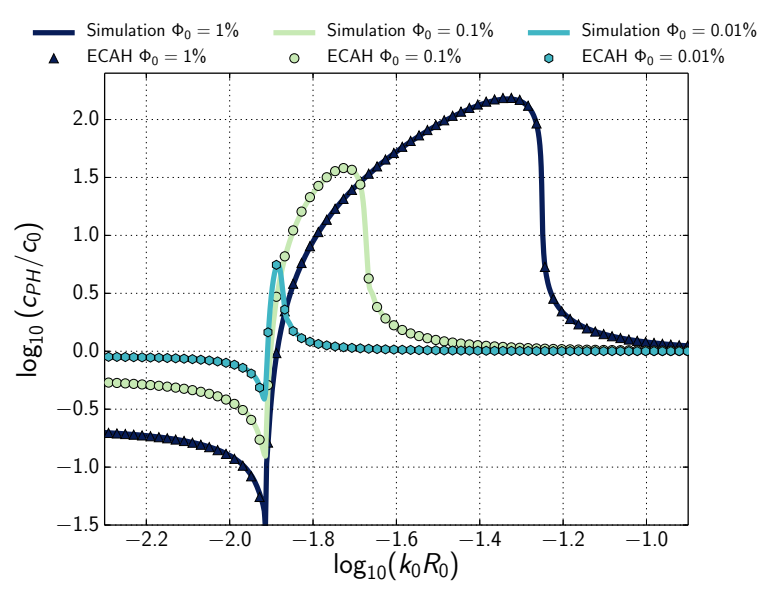

(a)

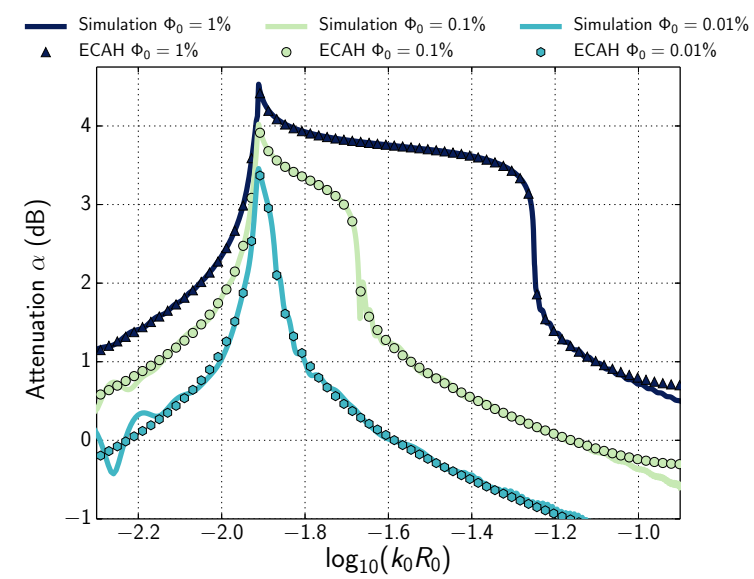

(b)

Fig. 3: Comparison between simulation and ECAH theory on the phase velocity (a) and attenuation (b) for several values of the volume fraction $\Phi_{0}$.

It is well-known that for small enough concentrations coherent waves appear in the effective medium. These waves correspond to the average, over all possible locations of the bubbles, of the multiply-scattered field in the actual host medium. The coherent wave propagation is governed by a complex effective wave number $K(\omega)$, which is a frequency-dependent function. The real part of the wave number is related to the phase velocity, and the imaginary part represents the attenuation. The effective wavenumber describing the sound propagation in a bubbly liquid is given by Waterman et al 26]. Details of the method used to calculate phase velocity and attenuation from numerical computations are given in Appendix A. It is possible to use the imaginary part to define the elastic mean free path $l_{e}=1 / 2 \operatorname{Im}[K]$, which can be interpreted as the propagation distance on which the wave remains coherent 27]. Based on this consideration, the signal is sampled at $x=2 \lambda_{0}$, in order to respect the condition $x<l_{e}$.

Fig. 3 compares the computed phase velocity and attenuation to the theoretical prediction for several values of the volume fraction $\Phi_{0}$. The agreement between the ECAH theory and the simulation is quantitative good in a wide range of frequencies and volume fractions. The significant variations of the phase velocity and attenuation are correctly described by the model. The choice of the numerical methods used to solve the hydrodynamical model (Eq. (3p) has been shown to be highly relevant. This result validates the present model as well as the numerical resolution method associated.

As in previous studies, below the resonant frequency of 


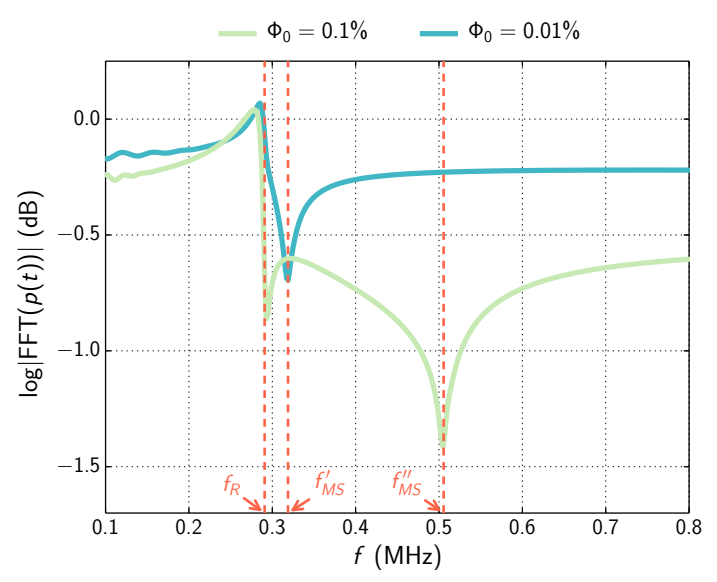

Fig. 4: Spectra of the total pressure signals simulated at $x=2 \lambda_{0}$ for two values of volume fraction $\Phi_{0} . f_{R}$ represents the Minnaert frequency for a bubble radius $R_{0}=10^{-5} \mathrm{~m} . f_{M S}^{\prime}$ and $f_{M S}^{\prime \prime}$ correspond to the cut off frequency of the bubbly medium for respectively $\Phi_{0}=0.1 \%$ and $\Phi_{0}=0.01 \%$.

bubbles $\left(\log _{10}\left(k_{0} R_{0}\right)<-1.9\right)$, the increase of the volume fraction strongly slows down the phase velocity. Beyond the Minnaert frequency $\left(\log _{10}\left(k_{0} R_{0}\right)>-1.9\right)$, an area of strong dispersion and attenuation is localized. This zone is enlarged as the fraction volume increases. As the wavelength approaches the bubble radius, the phase velocity tends towards the wave celerity in the fluid phase.

Fig. 4 depicts the spectra of the total pressure signals recorded. These results are obtained by centering the Gaussian pulse in a Hanning window by zero padding. Note that due to the high characteristic pulse frequency, the Fourier transform of the initial pressure disturbance is a Gaussian that can be approximated by constant energy source in the Fourier space for the range of frequencies of interest. Thus, the spectral signal amplitude depicted in Fig. 4 can be seen as the frequency response function of the bubbly medium. If we refer to Fig. 3, we can verify that the maximum attenuation range is bounded by two frequencies which correspond to the minimum observed on spectra of the total pressure signals in Fig. 4. The identification of these two frequencies is discussed in the following section.

\subsection{Resonance spectrum}

When investigating multiple scattering systems it is accepted that the coherent wave only appears after ensemble averaging. The coherent wave is distinguished from the ballistic wave, defined as the first well-defined pulse that crosses the bubbly medium. The ballistic wave can be measured on every realization of disorder and it corresponds to the very beginning of the signal (see Fig. 1). Similar to the incoming/incident wave, it is followed by a random signal commonly called "coda" 27. The coda waves can be seen as a speckle pattern in time which is highly sensitive to changes in the medium.

In this section we follow the classical experimental protocols used for the investigation of multiple scattering systems where the temporal signal is treated before analysing it by Fourier transform. Following these protocols, the data signal is filtered in order to remove the ballistic wave, so that the Fourier transform is applied only to the coda in order to obtain the so-called resonance spectrum 28 . Fig. 5 shows the modulus and phase of spectra for the resonance signals. For the two concentrations of bubbles tested here, one can observe that the pressure signal is composed of two predominant
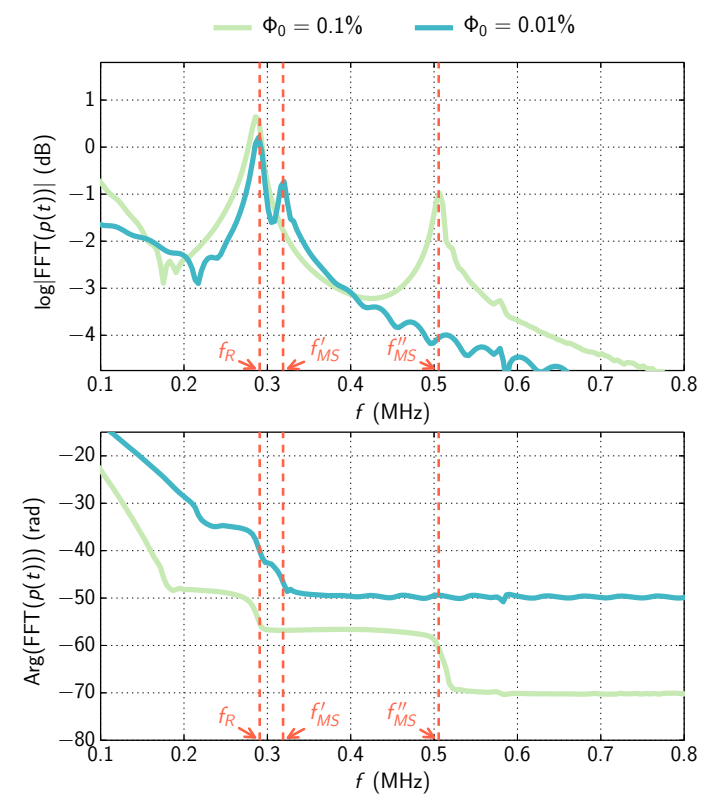

Fig. 5: Spectra (modulus and unwrapped phase) of the scattered signals simulated at $x=2 \lambda_{0}$ for two values of volume fraction $\Phi_{0}$. $f_{R}$ represents the Minnaert frequency for a bubble radius $R_{0}=10^{-5} \mathrm{~m}$. $f_{M S}^{\prime}$ and $f_{M S}^{\prime \prime}$ correspond to the cut off frequency of the bubbly medium for respectively $\Phi_{0}=0.1 \%$ and $\Phi_{0}=0.01 \%$.

frequencies (represented as dashed lines calculated according to the parameters used in the model). The first one, denoted $f_{R}$, corresponds to the Minnaert frequency, which obeys the classical expression [21]:

$$
f_{R}=\frac{1}{2 \pi} \sqrt{\frac{3 \kappa P_{g e}}{\rho R_{0}{ }^{2}}-\frac{2 \sigma}{\rho_{0} R_{0}{ }^{3}}} .
$$

The second frequency, denoted in the following as $f_{M S}$, corresponds to a cut off frequency that appears to be highly dependent on the volume fraction of bubbles. In general, the value of $f_{M S}$ increases with the concentration $\Phi_{0}$. This observation holds irrespective of the sampling position in the medium. We note that at both, $f_{R}$ and $f_{M S}$, we observe a phase jump that reveals that both frequencies are related to a resonance mechanism (Fig. 5 ).

The physical meaning of the resonance effect is obvious for the Minnaert frequency (Eq. 8). In order to interpret $f_{M S}$ we use the equivalent wavenumber of the bubbly medium, which is determined by harmonic balance following the procedure proposed by Hamilton and Blackstock [16. In the linear regime, only the generation of the first harmonic is considered (denoted by the index 1 ). The acoustic dimensionless pressure, the normalized mean velocity and the bubble radius can be approximated in harmonic regime as follows:

$$
\begin{aligned}
& P(x, t)=P_{1}(x) e^{\mathrm{j} \omega t}, \\
& Q(x, t)=Q_{1}(x) e^{\mathrm{j} \omega t}, \\
& R(x, t)=R_{1}(x) e^{\mathrm{j} \omega t} .
\end{aligned}
$$

Substitution of Eqs. (9), 10) and (11) into Eqs. (3) and (2) gives the following wave equation:

$$
\left(\nabla^{2}+K^{2}\right) P_{1}=0
$$

where the effective wavenumber is given by

$$
K^{2}=\frac{\omega^{2}}{c_{0}^{2}}\left(1-\Phi_{0}\right)+\frac{3 \Phi_{0}}{R_{0}^{2}}\left(\frac{\omega^{2}}{\omega_{R}^{2}-\omega^{2}+\mathrm{j} \delta \omega^{2}}\right)
$$

with $\omega_{R}=2 \pi f_{R}$ and $\delta=4 \mu /\left(\rho_{0} \omega R_{0}^{2}\right)$. 


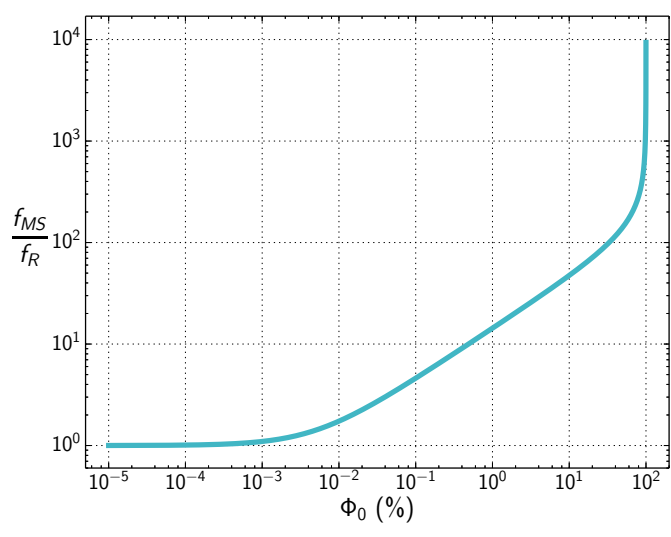

Fig. 6: Ratio between $f_{R}$ and $f_{M S}$ according to the volume fraction $\Phi_{0}$

If the effect of viscosity is neglected, the equivalent wavenumber of the bubbly medium tends to zero for the particular frequency

$$
f_{M S}=\frac{1}{2 \pi}\left(\sqrt{\omega_{R}^{2}+\frac{3 \Phi_{0}}{R_{0}{ }^{2}} \frac{c_{0}{ }^{2}}{\left(1-\Phi_{0}\right)}}\right) .
$$

One can clearly observe in Figs. 4 and 5 that this frequency predicts well the cut-off frequency beyond which the wave propagation is not altered by the bubbly medium in the linear regime.

Fig. 6 shows the evolution of the second frequency $f_{M S}$ as a function of the volume fraction $\Phi_{0}$. For highly diluted bubbly liquids, $f_{M S}$ tends to recover the Minnaert frequency. When increasing the void fraction $\Phi_{0}$, the difference between these two frequencies increases exponentially. Note that the concentration $\Phi_{0}$ can be derived from Eq. 14 as soon as we know the Minnaert frequency and the cut-off frequency $f_{M S}$. The dependency of the second frequency with the volume fraction invites us to think that $f_{M S}$ can be interpreted as a multiple scattering effect in the bubbly medium.

To sum up the main conclusions from the analysis of the numerical results in the linear regime, the Minnaert frequency $f_{R}$ and the cutoff frequency $f_{M S}$ appear naturally when performing the Fourier transform of the full pressure wave signal propagating in bubbly liquids. In order to observe them, the propagation distance of the pulse should be smaller than the elastic mean free path, which can be interpreted as the distance over which the coherent wave propagates 27 . The characteristic resonance frequency depends on the concentration and therefore can be interpreted as the result from the overall constructive interferences between the waves scattered in the medium. Making an analogy with periodic media, $f_{M S}$ can be regarded as a Bragg-type resonance associated to Bloch waves [26] given that it is a phenomenon resulting from the collective response of all the individual scatterers included in the medium. The physical meaning of $f_{R}$ is different. The Minnaert frequency $f_{R}$ does not depend on the concentration, it is a pole of the scattering coefficient of the bubble corresponding to the mode of vibration $n=0$. This is identified with a local resonance of Fano-type [26]. This observation is important for the analyses in the weakly nonlinear regime, as one can expect to observe different nonlinear behavior for $f_{R}$ and $f_{M S}$ when the excitation amplitude increases. (a)

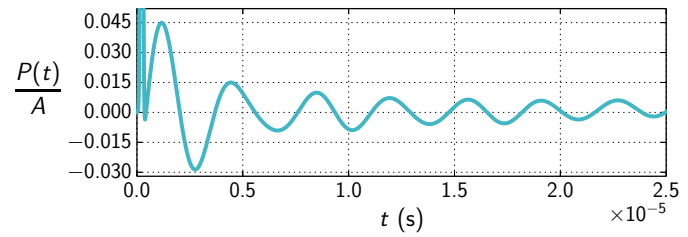

(b)

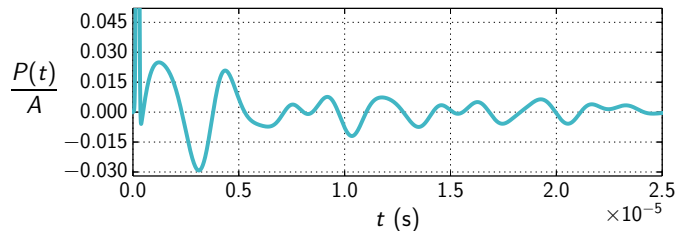

(c)

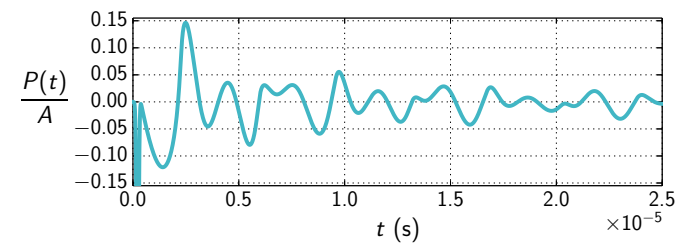

Fig. 7: Temporal evolution of the diffuse acoustic field produced by the bubbly medium. Comparison for different values of the initial pulse amplitude $A$. The dimensionless pressure $P(t)$ is normalized by $A$. (a) $A=10^{-5}$, (b) $A=0.9$ and (c) $A=-0.9$.

\section{Hardening and softening effects in bubbly liquids}

In order to study wave propagation for nonlinear bubbles dynamics, the numerical experiment presented in Section IV is carried out for different initial pulse amplitudes $A$ (see Eq. (7)). Two different type of excitations are tested. Compression tests $(A>0)$ and tension test $(A<0)$.

Fig. 7 shows the temporal evolution of the acoustic pressure $P(t)$ normalized by the amplitude of the initial pressure disturbance. One can clearly observe that the bubbly medium produces different acoustical responses according to the amplitude $A$ and its sign. When the pressure disturbance compresses the bubbles, $A>0$, the pulse amplitude alter the frequency content of the coda without affecting significantly their relative amplitude (see Figs. 7(a) and 7(b)). When the pulse amplitude has a negative amplitude, $A<0$, Fig. 7(c) shows that the nonlinear bubble response induces an intense acoustic field producing a complex and harmonically rich acoustic field. The difference observed between Figs. 7(b) and 7 (c) can be explained by the highly non-symetric response predicted by the Rayleigh-Plesset equation when bubbles expand with respect to situations when bubbles are compressed.

The amplitude-dependent effects are analyzed using the spectra of the total acoustic pressure as a function of the amplitude of the initial Gaussian pulse for positive compression pulses (Fig. 8) and negative pulses (Fig. 9) We define the frequency of the attenuation peak, $f_{ \pm}$, as the frequency for which we obtain the minimum modulus of the spectrum. This frequency corresponds to the value of $f_{M S}$ for low pulse amplitudes. When increasing the forcing amplitude we observe that this frequency is shifted.

When compression occurs, one observes a downward resonance frequency shift on $f_{M S}$ and a decrease of the quality factor when increasing the excitation amplitude. To the best of our knowledge, this phenomenon previously identified in sandstones or cracked materials 30, 31, has not been described in bubbly liquids yet. There are still some differences between the response of these systems. 


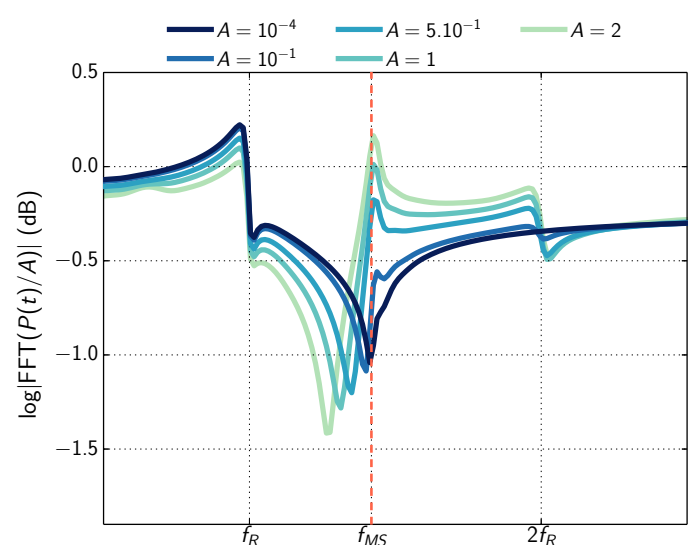

Fig. 8: Spectra of the total pressure signal simulated at $x=2 \lambda_{0}$ for different positive values of the initial pressure disturbance amplitude A. $f_{R}$ represents the Minnaert frequency for a bubble radius $R_{0}=10^{-5} \mathrm{~m}$. $f_{M S}$ corresponds to the cut-off frequency of the bubbly medium for $\Phi_{0}=0.05 \%$.

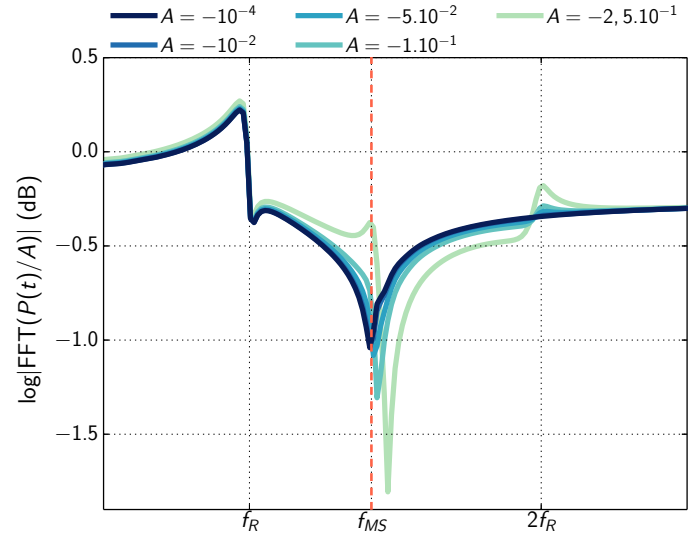

Fig. 9: Spectra of the total pressure signal simulated at $x=2 \lambda_{0}$ for different negative values of the initial pressure disturbance amplitude $A . f_{R}$ represents the Minnaert frequency for a bubble radius $R_{0}=10^{-5} \mathrm{~m}$. $f_{M S}$ corresponds to the cut-off frequency of the bubbly medium for $\Phi_{0}=0.05 \%$.
Indeed, because the resonance curve is nonsymmetric, the amplitude-dependent quality factor cannot be estimated precisely by measuring the width of the resonance curve at half amplitude (or $-3 \mathrm{~dB}$ ). The downward frequency shift can be interpreted as an amplitude-dependent modification of the acoustic wave velocity or an average softening of the medium 32. In the case of granular media, when softening is observed, it has been shown that frictional nonlinearities are caused by the mechanical behavior of the contacts between elastic beads lead to higher levels of friction than those encountered in homogeneous bulk media 33 . In our case, there is no direct contact between bubbles but they interact through the influence of the bubbles in the averaged pressure field. This effect makes nonlinear response of the mode associated to $f_{M S}$ different from the classical scenario discussed in granular media.

The response of the system in the weakly nonlinear regime turns out to be different depending on the sign of the initial Gaussian pulse. Thus, when a negative amplitude is imposed (see Fig. 9), we observe that resonance frequencies shift to high frequencies when increasing the amplitude. This particular behavior, usually referred to as "hardening of the medium" in granular systems [34, 35, 36, shows that in a bubbly liquid it suffices to reverse the sign of the amplitude to shift from hardening to softening. The nonlinear dynamics of bubbles together with the multiple scattering effects may be behind this particular nonlinear response.

Fig. 10 represents the shift between the theoretical value of $f_{M S}$ (Eq. 14 ) and the frequency $f_{ \pm}$at which the attenuation is maximal as a function of the amplitude of the initial pulse. The relative resonance frequency shift $\Delta f / f_{M S}=\left(f_{ \pm}-f_{M S}\right) / f_{M S}$ scales with the amplitude of the initial pulse according to a law to be determined. As the softening/hardening of the medium results in an amplitudedependent modification of the acoustic wave velocity, we try the simplest correction proposed for resonances in a nonlinear system that propose to modify the sound speed with an expression of the form 37 .

$$
c_{ \pm}(A)=c_{0}\left(1 \pm|A|^{m} / b\right),
$$

where $m$ and $b$ are adjustable parameters. To do this, we insert Eq. 15 into Eq. (14) to obtain

$$
f_{ \pm}(A)=\frac{1}{2 \pi} \sqrt{\omega_{R}^{2}+\frac{3 \Phi_{0}}{R_{0}^{2}\left(1-\Phi_{0}\right)} c_{ \pm}(A)^{2}} .
$$

This frequency can be now be fitted with the simulated data (dot) using the least squares minimization method as shown in Fig. 10. The good fitting between the model and numerical simulations seems to confirm the choice of the law introduced for the equivalent sound velocity in Eq. 15 for moderate amplitudes.

In order to gain further insight into the similarities of the response of bubbly liquids with respect to other media we assume now that $|A|$ is small enough. In this situation we know that an amplitude dependence close to $\Delta f / f_{M S} \propto$ $A^{2}$ is typical of Hertzian contacts (frictional nonlinearities) while an amplitude dependence close to $\Delta f / f_{M S} \propto A$ rather characterizes hysteretic quadratic nonlinearities [32, 33]. In our case, it follows directly from Eq. 16) and Eq. 15 that

$$
\frac{\Delta f}{f_{M S}}= \pm \frac{3 \Phi_{0}}{1-\Phi_{0}} \frac{c_{0}^{2}}{b R_{0}^{2} \omega_{M S}}|A|^{m} .
$$

This result shows that the relative resonance frequency shift $\Delta f / f_{M S}$ during compression $(m=1)$ seems to be characteristic of hysteretic quadratic nonlinearities 32 which 


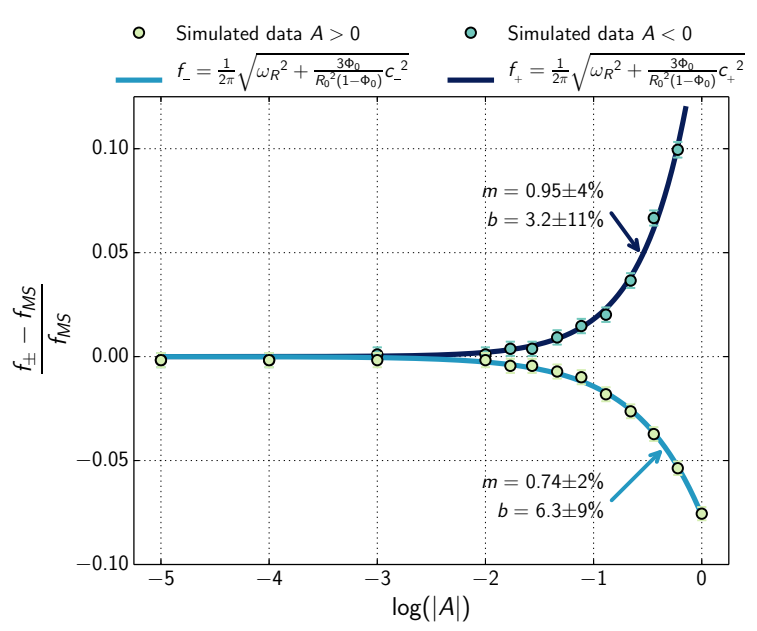

Fig. 10: Evolution of the maximum attenuation frequency according to the amplitude of the initial pressure disturbance. Dots represent the simulated data for positive and negative initial Gaussian pulses. Solid lines are calculated from the Eq. 16.

$$
R_{0}=10^{-5} \mathrm{~m} \text { and } \Phi_{0}=0.05 \% \text {. }
$$

also establish some similarities to the studies of acoustic hardening and optic softening of phonons 37.

It is worthy mentioning that although in this study we have decided to correct the effective sound velocity to keep the analogy with the hardening/softening effect in solids. It is also possible to fit the numerical results by using an effective concentration $\Phi_{ \pm}(A)$ that allows us to define $f_{ \pm}(A)$ as

$$
f_{ \pm}(A)=\frac{1}{2 \pi} \sqrt{\omega_{R}^{2}+3 \frac{c_{0}^{2}}{R_{0}^{2}} \frac{\Phi_{ \pm}(A)}{1-\Phi_{ \pm}(A)}} .
$$

This correction has the same effect on the frequency shift than the correction of the sound speed if the dependence of the effective void fraction with the excitation amplitude has the following form

$$
\Phi_{ \pm}(A)=\Phi_{0}\left(1 \mp|A|^{m} / B\right) .
$$

Assuming that $|A|$ is small enough we exactly recover Eq. (17) when $B=-b / 2$. Thus, we can also interpret the softening and hardening effects as net change on the effective void fraction. When compression occurs, the nonlinear response of the bubbles make the bubble compression more important than the bubble expansion, reducing the effective void fraction and inducing an apparent decrease on the effective sound velocity. The opposite situation occurs when the pressure wave induce tension: the bubble expansion is privileged increasing the effective void fraction of the medium which at the same time can be seen as an apparent increase of the sound velocity.

\section{Acoustic harmonic generation in bubbly liquids}

In this section we focus our attention in the appearance of harmonics in the resonance spectrum 28, which is obtained for several positive amplitudes of the initial Gaussian pulse (Fig. 11). For low amplitudes (dashed line) the resonance spectrum of the scattered field contains two predominant frequencies, as observed previously for linear regime in Fig. 5 When the amplitude grows up (solid lines), the harmonics of the Minnaert frequency progressively appear. Figs. 8 and 9 already showed a relatively weak Minnaert harmonic on the full spectrum of the pressure signal. However, the intensity of these harmonics is significantly increased in the resonance spectrum (Fig. 11). This effect, directly related to the removal

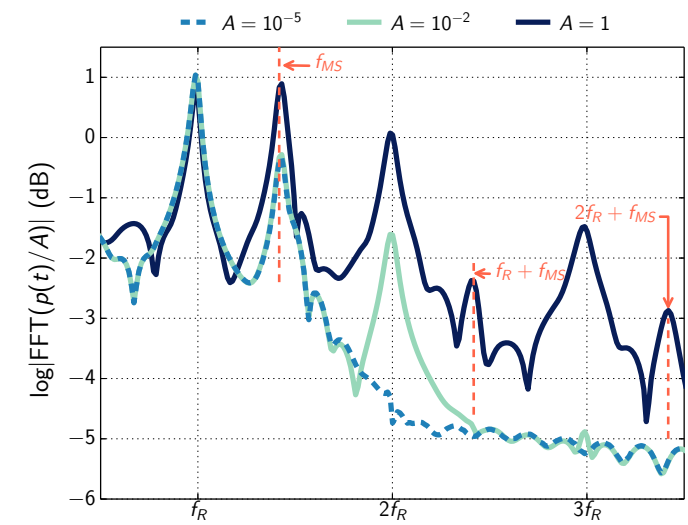

Fig. 11: Module spectra of the diffuse part to pressure signals for $\Phi=0.05 \%$ (normalized by $A$ ), comparison according to the initial pulse amplitude $A . f_{R}$ represents the Minnaert frequency for a bubble radius $R_{0}=10^{-5} \mathrm{~m}$. $f_{M S}$ corresponds to the frequency of the second minimum of group velocity.

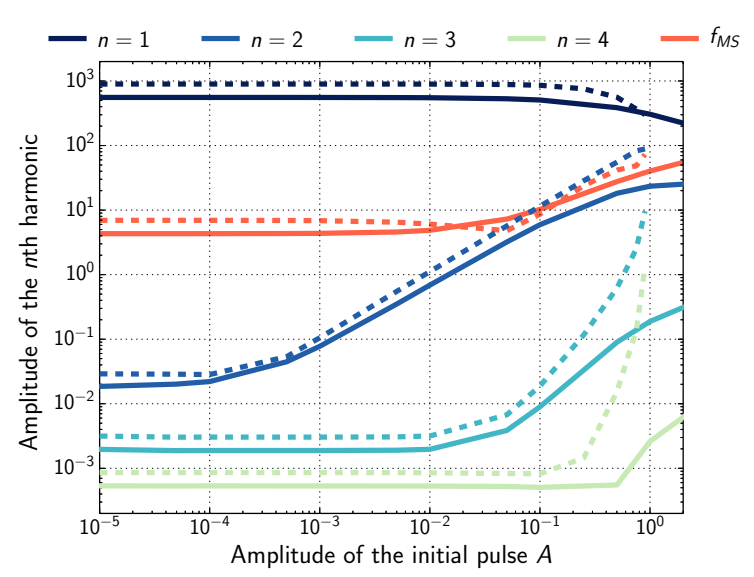

Fig. 12: Amplitude evolution of the harmonic components of diffuse part to the pressure signals as a function of the amplitude of the initial pulse $\left(\Phi=0.05 \%\right.$ and $\left.R_{0}=10^{-5} \mathrm{~m}\right)$. Solid and dashed lines represent simulations for positive and negative pulses respectively.

of the ballistic signal from the total pressure, is interpreted by the fact that the Minnaert harmonics result from a cumulative effect well known in non linear acoustics [16].

The appearance of softening-hardening effects in the full and resonance spectrum is significantly different. In this case, softening-hardening effects are less clearly seen in the resonance spectrum. This can be explained by the great part of the coherent wave energy contained in the ballistic wave. Removing the ballistic part of the wave weaken the contribution of the coherent multiple scattering reducing also the softening-hardening phenomenon related to the coherent waves. Conversely, when the ballistic wave is removed the information contained in the coda is highlighted. As the time duration of the coda is much longer than that of the ballistic wave, it becomes easy to detect harmonics due to cumulative effects (Fig. 11). Based on these results we conclude that the ballistic wave basically contains all the information related to softening-hardening effects, whereas the coda include most of the nonlinear harmonic content of the signal.

Another interesting remark derived from the analysis of the resonance spectrum is that for amplitudes of the same order of magnitude to the static pressure $(A \simeq 1)$ we observe that Minnaert harmonics and the cut off frequency $f_{M S}$ are mixed. This is yet another example of the combination between nonlinearity of bubble dynamics with the multiple scattering phenomenon. One can also notice that harmonics related to $f_{M S}$ are not detected. This is not surprising. 
As previously discussed we refer here to the analogy with Bragg-type resonances, $f_{M S}$ seems to be a consequence of the multiple scattering phenomena that take place throughout the bubbly medium, thereby excluding the possibility of cumulative effects closely linked to a characteristic distance of propagation in the medium.

In order to characterize harmonic generations, Fig. 12 shows the amplitude evolution of harmonics according to the amplitude of the initial pulse $A$. One can observe that the amplitude of the Minnaert harmonics appear for negative pulses (dashed lines) at lower amplitudes than in the case of positive pulses (solid lines). Close to the static pressure $(A \simeq 1)$, the harmonics level is clearly greater for negative pulses than for positive pulses. This amplitude evolution of harmonic components is typical to nonlinear mechanisms.

Fig. 12 also represents the amplitude evolution of the multiple scattering frequency $f_{M S}$. The amplitude of this frequency can equally vary according to the amplitude of the initial pressure disturbance. Compared to the harmonics of Minnaert frequency, the amplitude of $f_{M S}$ increases for stronger initial pulses around to $A>5 \cdot 10^{-2}$. Note that, the softening effects appear from such amplitudes. Nonlinear multiplescattering effects seem to be produced when the bubbles have a rich-harmonically motion (when the 3rd harmonic is generated.).

\section{Conclusion}

The linear and weakly nonlinear propagation of acoustic waves in monodisperse bubbly liquids is numerically investigated using a hydrodynamic model based on the averaged two-phase fluid equations coupled to the RPNNP equation to model the bubble dynamic response. The numerical method used to solve the problem is based on a finite difference approach coupled with an ODE solver for the RPNNP equation. To study the wave dispersion in bubbly liquids, a Gaussian pulse is imposed for the initial pressure disturbance. The present model is validated in the linear regime by comparing the phase velocity and attenuation of the coherent wave computed by the model with the results obtained by the ECAH multiple scattering theory.

In the linear regime, the analysis of the pressure signals reveals the appearance of two resonance frequencies. In addition to the Minnaert frequency $f_{R}$, another resonance frequency $f_{M S}$ is identified which is sensitive to the bubble concentration. This frequency, associated to multiple scattering effects, is clearly observed when the propagation distance of the pulse is smaller than the elastic mean free path, defined as the distance over which the coherent wave propagates. In practice, this distance corresponds to a few wavelengths. If one tries to make an analogy with periodic media, the Minnaert frequency $f_{R}$ corresponds to the resonance frequency of an Helmoltz-type resonator whereas the multiple scattering resonance $f_{M S}$ to a Bragg-type resonance. The first is a local resonance of Fano-type while the second is a frequency due to overall constructive interferences between the waves scattered in the medium.

When the amplitude of the initial pressure disturbance becomes of the order of the static pressure various nonlinear effects become clear. The generation of higher harmonics is observed for $f_{R}$ whereas we do not observe any harmonics related to $f_{M S}$. Instead we observe linear combinations between the Minnaert harmonics and $f_{M S}$ in the following form $m f_{R}+f_{M S}$, with $m$ a positive integer. This can be interpreted as an example of interaction between nonlinear effects and multiple scattering phenomena.
In any case, the most notorious effect is certainly the identification of softening-hardening phenomena related to the multiple scattering resonance $f_{M S}$. Interestingly, the resonance frequency $f_{M S}$ shifts to lower frequencies when increasing the positive excitation amplitudes (cf. Fig. 8) while $f_{M S}$ shifts to high frequencies when increasing the negative excitation amplitude (cf. Fig. 9). In both cases, a decrease of the quality factor is observed when increasing the excitation amplitude. To the best of our knowledge this phenomenon, frequent in sandstones or cracked materials, has not been observed in bubbly liquids yet. We show that the frequency shift can be explained assuming that the acoustic wave velocity depends on the excitation amplitude according to a law given in Eq. 15. At the same time, we show that the relative resonance frequency shift obey a law which is more complex than those usually encountered for sandstones or cracked materials (cf. Eq. (17)).

In perspective to this work, it would be interesting to study whether other bubbly media, like those containing UCAs, may influence the emergence of the nonlinear effects analyzed in this article.

\section{Acknowledgements}

The authors acknowledge funding from the French National Agency for Research (ANR-11BS09-007-03) project DiAMAN.

\section{A Computation of phase velocity and atten- uation}

Phase velocity and attenuation are computed following Ando et al. 12. Consider a plane wave formed as $e^{\mathrm{j}(2 \pi f t-K x)}$, where $\mathrm{j}$ is the imaginary unit and $K$ the complex wave number of the equivalent medium. The real and imaginary parts of $K$ can be determined based on the time history of the acoustical pressure sampled at two different locations, denoted as $p\left(x_{1}, f\right)$ and $p\left(x_{2}, f\right)$. Its discrete Fourier transform coefficients can be written as

$$
\hat{p}(x, f)=\hat{p}_{0} e^{-\mathrm{j} k_{r}(f) x} e^{-k_{i}(f) x},
$$

where $k_{r}$ and $k_{i}$ are respectively the real and the complex part of the complex wave number. Given the complex ratio $P_{1 / 2}=$ $\hat{p}\left(x_{1}, f\right) / \hat{p}\left(x_{2}, f\right)$, the complex wave number is computed as

$$
\begin{gathered}
k_{r}=\frac{1}{\Delta x_{1 / 2}} \cos ^{-1}\left(\operatorname{Re}\left[P_{1 / 2}\right] e^{-\ln \left|P_{1 / 2}\right|}\right), \\
k_{i}=\frac{1}{\Delta x_{1 / 2}} \ln \left|P_{1 / 2}\right|
\end{gathered}
$$

with $\Delta x_{1 / 2}=x_{2}-x_{1}$.

Thereby, the phase velocity $c_{\mathrm{PH}}$ and attenuation $\alpha$ are defined [10] as:

$$
\begin{gathered}
c_{\mathrm{PH}}=\frac{\omega}{k_{r}}, \\
\alpha=\log \left(k_{i}\right) .
\end{gathered}
$$

\section{References}

[1] E. Stride and N. Saffari, "Investigating the significance of multiple scattering in ultrasound contrast agent particle populations," IEEE Transactions on 52(12), 2332-2345 (2005)

[2] M. Tang and R. J. Eckersley, "Nonlinear propagation of ultrasound through microbubble contrast agents and implications for imaging," IEEE Transactions on 53(12), 2406-2415 (2006). 
[3] M.-X. Tang, J. Loughran, E. Stride, D. Zhang, and R. J. Eckersley, "Effect of bubble shell nonlinearity on ultrasound nonlinear propagation through microbubble populations," J. Acoust. Soc. Am. 129(3), EL76-EL82 (2011).

[4] L. Demi, H. Wijkstra, and M. Mischi, "Cumulative phase delay between second harmonic and fundamental components-A marker for ultrasound contrast agents," J. Acoust. Soc. Am. 136(6), 2968-2975 (2014).

[5] M. Cavaro, C. Payan, J. Moysan, and F. Baqué, "Microbubble cloud characterisation by nonlinear frequency mixing," J. Acoust. Soc. Am. 129(5), EL179-EL183 (2014).

[6] V. Leroy, A. Strybulevych, M. G. Scanlon, and J. H. Page, "Transmission of ultrasound through a single layer of bubbles," Eur. Phys. J. B Soft 29(1), 123-130 (2009).

[7] X. Guo, Z. Lin, J. Tu, B. Liang, J. Cheng, and D. Zhang, "Modeling and optimization of an acoustic diode based on micro-bubble nonlinearity," J. Acoust. Soc. Am. 133(2), 1119-1125 (2013).

[8] L. L. Foldy, "The multiple scattering of waves. Part I: general theory of isotropic scattering by randomly distributed scatterers," Phys. Rev. 6719, 107-119 (1945).

[9] E. L. Carstensen and L. L. Foldy, "Propagation of sound through a liquid containing bubbles," J. Acoust. Soc. Am. 19, 481-501 (1947)

[10] K. W. Commander and A. Prosperetti, "Linear pressure waves in bubbly liquids: Comparison between theory and experiments," J. Acoust. Soc. Am. 85, 732-746 (1989).

[11] D. Zhang and A. Prosperetti, "Momentum and energy equations for disperse two-phase flows and their closure for dilute suspensions," Int. J. Multiphase. Flow. 23 425-453 (1997).

[12] K. Ando, T. Colonius, and C. E. Brennen, "Numerical simulation of shock propagation in a polydisperse bubbly liquid," Int. J. Multiphase Flow 37(6), 596-608 (2011).

[13] L. van Wijngaarden, "On equations of motion for mixtures of liquid and gas bubbles," J. Fluid Mech. 33, 465-474 (1968).

[14] R. E. Cafisch, M. J. Miksis, G. C. Papanicolaou, and L. Ting, "Effective equations for wave propagation in bubbly liquids," J. Fluid Mech. 153, 259-273 (1985).

[15] F. Coulouvrat, J.-L. Thomas, K. Astafyeva, N. Taulier, J.-M. Conoir, and W. Urbach, "A model for ultrasound absorption and dispersion in dilute suspensions of nanometric contrast agents," J. Acoust. Soc. Am. 132(6), 3748-3759 (2012).

[16] M. F. Hamilton and D. T. Blackstock, Nonlinear Acoustics (Academic Press, 1998), chapter 5.

[17] D. Fuster and F. Montel, "Mass transfer effects on linear wave propagation in diluted bubbly liquids," J. Fluid Mech. 779, 598-621 (2015).

[18] A. T. Preston, T. Colonius, and C. E. Brennen, "A reduced-order model of diffusive effects on the dynamics of bubbles," Physics of Fluids 19, 123302 (2007).

[19] D. Fuster and T. Colonius, "Modelling bubbles clusters in compressible liquids," J. Fluid Mech. 688, 352-389 (2011).
[20] D. Fuster, J.-M. Conoir, and T. Colonius, "Effect of direct bubble-bubble interactions on linear-wave propagation in bubbly liquids," Phys. Rev. E 90(6), 063010, 2014.

[21] T. Leighton, The Acoustic bubble (Academic Press, 1994), pp. 301-312.

[22] J.-P. Berenger, "A perfectly matched layer for the absorption of electromagnetic waves," J. Comput. Phys. 114(2), 185-200 (1994).

[23] R. E. Challis, M. J. W. Povey, M. L. Mather, and A. K. Holmes, "Ultrasound techniques for characterizing colloidal dispersions," Rep. Prog. Phys. 68(7), 1541-1637 (2005).

[24] T. Valier-Brasier, J.-M. Conoir, F. Coulouvrat, and J.-L. Thomas, "Sound propagation in dilute suspensions of spheres: Analytical comparison between coupled phase model and multiple scattering theory," J. Acoust. Soc. Am. 138(4), 2598-2612 (2015).

[25] V. V. Kuznetsov, V. E. Nakoryakov, B. G. Pokusaev, and I. R. Shreiber, "Propagation of perturbations in a gas-liquid mixture," J. Fluid Mech. 85(1), 85-96 (1978).

[26] P. C. Waterman and R. Truell, "Multiple Scattering of Waves," J. Math. Phys 2(4), 512-537 (1961).

[27] A. Derode, A. Tourin, and M. Fink, "Random multiple scattering of ultrasound. I. Coherent and ballistic waves," Phys. Rev. E 64(3), 036605 (2001).

[28] N. D. Veksler, Resonance Acoustic Spectroscopy (Springer-Verlag, Berlin, Heidelberg 1993), pp. 1-282.

[29] G. Theocharis, O. Richoux, V. Romero Garcia, A. Merkel, and V. Tournat, "Limits of slow sound propagation and transparency in lossy, locally resonant periodic structures," New Journal of Physics 16(9), 093017 (2014).

[30] P. A. Johnson, B. Zinszner, and P. Rasolofosaon, "Resonance and elastic nonlinear phenomena in rock," Journal of Geophysical Research 101(B5), 11553-11564 (1996).

[31] A. Novak, M. Bentahar, V. Tournat, R. El Guerjouma, and L. Simon, "Nonlinear acoustic characterization of micro-damaged materials through higher harmonic resonance analysis," NDT\&E International 45, 1-8 (2012).

[32] J. Cabaret, V. Tournat, and P. Béquin, "Amplitudedependent phononic process in a diatomic granular chain in the weakly nonlinear regime," Phys. Rev. E 86, 041305 (2012).

[33] L. Bonanomi, G. Theocharis, and C. Daraio, "Wave propagation in granular chains with local resonances," Phys. Rev. E 91, 033208 (2015).

[34] D. L. Johnson, H. A. Makse, N. Gland, and L. Schwartz, "Nonlinear elasticity of granular media," Physica B $\mathbf{2 7 9}$ 134-138 (2000).

[35] S. Job, F. Santibañez, F. Tapia, and F. Melo, "Nonlinear waves in dry and wet Hertzian granular chains," Ultrasonics 48(6), 506-514 (2008).

[36] T. Brunet, X. Jia, and P. Mills, "Mechanisms for acoustic absorption in dry and weakly wet granular media," Phys. Rev. Lett. 101(13), 138001 (2008). 
[37] C. Q. Sun, L. K. Pan, C. M. Li, and S.Li, "Size-induced acoustic hardening and optic softening of phonons in InP, $\mathrm{CeO} 2$, SnO2, CdS, Ag, and Si nanostructures," Phys. Rev. B 72, 134301 (2005). 